\title{
Qualidade de vida de pacientes acometidos por acidente vascular cerebral
}

\author{
Quality of life of patients affected by cerebral vascular accident
}

Francisco Valter Miranda Silva ${ }^{1^{*}}$

Orcid: https://orcid.org/0000-0002-7122-2466

Claudiana Batista de Brito ${ }^{3^{*}}$

Orcid: https://orcid.org/0000-0002-1827-8276

Elizandra Menezes Maia ${ }^{5^{*}}$

Orcid: https://orcid.org/0000-0002-8051-7645

Wênia Sâmia Bandeira Ferreira ${ }^{\text {* }}$

Orcid: https://orcid.org/0000-0003-2744-6486
Ana Beatriz Castro Oliveira ${ }^{2 *}$

Orcid: https://orcid.org/0000-0002-2220-8764

Franklin Douglas Saboia de Sousa ${ }^{4 *}$

Orcid: https://orcid.org/0000-0002-7601-8632

João Victor Pereira da Silva ${ }^{6 *}$

Orcid: https://orcid.org/0000-0002-5863-8109

Paula Pessoa de Brito Nunes ${ }^{8 *}$

Orcid: https://orcid.org/0000-0002-5189-8469

\begin{abstract}
Resumo
O Acidente Vascular Cerebral (AVC) é uma doença grave, suas sequelas podem comprometer a qualidade de vida das pessoas acometidas. Objetivo: Analisar a qualidade de vida de pacientes acometidos por AVC. Metodologia: Trata-se de um estudo descritivo, exploratório do tipo transversal, com amostra composta por pacientes acometidos por AVC de ambos os sexos, com idade acima de 21 anos. Foram aplicados os questionários: Mini-Exame do estado mental (MEEM), Sociodemográfico e o Medical Outcomes Short-Form 36-item Health Survey (SF-36). Resultados: 20 pacientes estavam em atendimento, desses apenas 15 atingiram a pontuação necessária no MEEM para entrar na pesquisa. $9(60,0 \%)$ eram do sexo masculino e $6(40,0 \%)$ do sexo feminino, com idade igual ou superior a 60 anos, com faixas etárias de 60 a 69 anos $6(40,0 \%)$ e 71 a 79 anos $2(13,3 \%)$, em relação à raça, a branca e a parda se igualaram com 7 (46,7\%), ao tipo de AVC observou-se que $11(73,3 \%)$ dos pacientes sofreram AVC Isquêmico e 4 (26,7\%) AVC Hemorrágico. Sobre o tempo de ocorrido do AVC, 12 (80\%) tinham tido há mais de 1 ano. Foram observados baixos escores em todos os domínios da SF36 , principalmente nos aspectos físicos $(26,66)$, capacidade funcional $(43,33)$, e aspectos emocionais $(41,93)$. Conclusão: Foi observado o comprometimento na percepção da Qualidade de Vida (QV) com valores baixos de todos os domínios, principalmente nos aspectos físicos, emocionais e capacidade funcional, com maior frequência em pacientes com diagnóstico clínico de AVC do tipo isquêmico, com igual ou superior a 60 anos de gênero masculino.

Palavras-chave: acidente vascular cerebral; qualidade de vida; fatores de risco.
\end{abstract}

\footnotetext{
${ }^{1}$ E-mail: valtermiranda15@gmail.com

${ }^{2}$ E-mail: obeaoliveira@gmail.com

${ }^{3}$ E-mail: cbbdiana@gmail.com

${ }^{4}$ E-mail: franklinsousa_@hotmail.com

${ }^{5}$ E-mail: elizandramenezesmaia@hotmail.com

${ }^{6}$ E-mail: viictorsilvaa13@gmail.com

${ }^{7}$ E-mail: wennisamiaa@gmail.com

${ }^{8}$ E-mail: paulanunes2912@gmail.com

* Centro Universitário Ateneu (UNIATENEU), Fortaleza, Ceará, Brasil.
} 


\begin{abstract}
Stroke is a serious disease, its sequelae can compromise the quality of life of the people affected. Objective: To analyze the quality of life of stroke patients. Methodology: This is a descriptive, exploratory cross-sectional study, with a sample composed of stroke patients of both sexes, aged over 21 years. The questionnaires were applied: Mini-Mental State Examination (MMSE), Sociodemographic and the Medical Outcomes Short-Form 36-item Health Survey (SF-36). Results: 20 patients were in attendance, only 15 of them reached the required MMSE to enter the research. $9(60.0 \%)$ were male and $6(40.0 \%)$ were female, aged over 60 years, aged from 60 to 69 years $6(40.0 \%)$ and 71 to 79 years $2(13.3 \%)$, in relation to race, white and brown were equal to $7(46.7 \%)$, for the type of stroke it was observed that $11(73.3 \%)$ of the patients suffered a stroke Ischemic and $4(26.7 \%)$ Hemorrhagic Stroke. About the time of the stroke, $12(80 \%)$ had had it for more than 1 year. Low scores were observed in all domains of the SF-36, mainly in the natural aspects (26.66), functional capacity (43.33), and emotional aspects (41.93). Conclusion: It was observed a compromise in the perception of Quality of Life (QOL) with low values in all domains, mainly in the physical, emotional and functional capacity, with a higher frequency in patients with a clinical diagnosis of ischemic stroke, aged over 60 years of male gender

Keywords: stroke; quality of life; risk factors.
\end{abstract}

\section{Introdução}

O Acidente Vascular Cerebral (AVC) é caracterizado por uma interrupção do fluxo sanguíneo, ou extravasamento de líquido para o encéfalo, que pode resultar em óbito, devido a um problema vascular, ocasionando infarto ou hemorragia do tecido neural, apresentando como consequência déficits nas funções neurológicas ${ }^{1}$.

Em sua etiologia, o AVC classificase de duas formas: Acidente Vascular Cerebral Hemorrágico (AVCh), que decorre de um extravasamento de sangue atingindo as estruturas do sistema nervoso central. Enquanto o Acidente Vascular Cerebral Isquêmico (AVCi) é decorrente da obstrução dos vasos, limitando o suprimento de oxigênio e substratos para o tecido cerebral ${ }^{2}$

Mundialmente, a patologia em questão apresenta elevadas taxas de incapacidades e morbimortalidades, sendo considerado um problema de saúde pública e a segunda maior causa de morte no mundo. De acordo com a Organização Mundial de Saúde (OMS, 2013), estima-se que até 2030 o AVC continue sendo a segunda maior causa de morte, atingindo cerca de 15 milhões de pessoas por ano e cerca de 5 milhões de óbitos. ${ }^{3}$ No Brasil, o
AVC é considerado uma doença grave, com taxa de sobrevida de $47 \%$, sendo considerado um problema de saúde pública por gerar incapacidade e na maioria dos casos perda da independência, o que implica diretamente na Qualidade de Vida (QV) . ${ }^{4}$

De acordo com a $\mathrm{OMS}^{5}$, a QV refere-se "à percepção do indivíduo de sua inserção na vida, no contexto da cultura e sistemas de valores nos quais ele vive e em relação aos seus objetivos, expectativas, padrões e preocupações". Logo, tudo que engloba desde a sua saúde física $\mathrm{e}$ psicológica, bem como as alterações no contexto individual, social e cultural em que esse indivíduo vive e que está exposto pode interferir em sua QV.

Nessa perspectiva, os indivíduos acometidos por AVC apresentam dificuldades em realizar suas Atividades de Vida Diária (AVD's), passando a depender de forma parcial ou total do auxílio de familiares ou cuidadores. Além disso, essa nova realidade pode desencadear estresse, ansiedade ou quadros depressivos em virtude das limitações impostas pela doença que implicam no convívio familiar e social, visto que os domínios da $\mathrm{QV}$ podem ser interferidos devido à redução funcionalidade causada pela doença 6,7 . Dessa forma, o AVC causa um grande 
impacto na vida das pessoas, seja em caráter individual, familiar ou social, pois atividades como trabalhar e lazer são prejudicadas, sendo desafiador o processo de reabilitação até sua reintegração na família e na sociedade.

Diante do exposto, considerando as limitações causadas pelo AVC, em que estas podem implicar em sua qualidade de vida, justifica-se a realização deste estudo. Além disso, houve o interesse em realizar essa pesquisa a partir de experiências vividas pelos pesquisadores em um centro especializado no cuidado de pacientes com $\mathrm{AVC}$, tornando-a relevante quanto à análise dos aspectos que alteram sua condição de saúde, podendo assim alertar e incentivar a promoção de saúde visando a melhora da QV da população estudada. Portanto, o objetivo do estudo foi analisar a QV de paciente acometidos por AVC.

\section{Materiais e Métodos}

\section{Tipo de estudo e amostra}

Trata-se de um estudo descritivo exploratório do tipo transversal, realizado nos meses de setembro e outubro de 2019, em duas clínicas-escolas localizadas no município de Fortaleza-Ceará, as clínicasescolas Centro Integrado de Saúde Ateneu (CISA) e do Projeto Qualivida realizam atendimentos ambulatórias de diferentes especialidades na área da saúde.

A mostra deste estudo se deu por conveniência, sendo recrutados para participar da pesquisa todos os pacientes diagnosticados AVC em atendimento no período supracitado nas referidas instituições.

Esta pesquisa foi aprovada pelo Comitê de Ética em Pesquisa do Centro Universitário Ateneu (UNIATENEU), Fortaleza-Ceará, com parecer de $n^{\circ}$. 3.506.088, em consonância com a Resolução 466/12 do Conselho Nacional de Saúde. Os participantes consentiram sua participação pela assinatura do Termo de Consentimento Livre e Esclarecido (TCLE).

\section{Delineamento da pesquisa}

Após as autorizações, os pacientes foram submetidos à coleta dos dados com a aplicação de três instrumentos de coleta, sendo os questionários aplicados, nas próprias clínicas após o atendimento sem que houvesse interferência na rotina de tratamento desses pacientes, em local reservado com o sigilo dos dados.

\section{Critérios de Inclusão e Exclusão}

Foram inclusos pacientes com diagnóstico clínico de AVC, com idade acima de 21 anos, de ambos os sexos, e que estavam em atendimento nas referidas instituições, no período da coleta que ocorreu nos meses de setembro e outubro de 2019.

Após o esclarecimento detalhado do estudo, foi solicitado que os indivíduos ou cuidador/acompanhante assinassem o TCLE. Foram excluídos da pesquisa aqueles com incapacidade de comunicação (afasia, dispraxia oral e dispraxia da fala, e a disartria), déficit cognitivo e visual grave que impeça a compreensão e os que não atingiram a pontuação de 20 pontos do Mini-Exame do Estado Mental (MEEM).

\section{Procedimentos}

Os instrumentos de coleta aplicados na primeira etapa foram:1) Mini-Exame do Estado Mental (MEEM), versão brasileira. 2) Questionário sociodemográfico, histórico de saúde e estilo de vida e 3) SF36 (Medical Outcomes Study 36 - Item Short- Form Health Survey).

O MEEM, serve para rastrear declínios cognitivos, demências e selecionar os pacientes que estavam aptos a responder os demais questionários, com ponto de corte de 20 pontos. O MEEM é um instrumento utilizado para a avaliação da função cognitiva, parâmetros quanto à orientação temporal e espacial, memória imediata, cálculo, linguagem e apraxia construtiva. A pontuação máxima é de 30 pontos e pode ser influenciada pela escolaridade do indivíduo ${ }^{8,9}$.

O Questionário sociodemográfico, histórico de saúde e estilo de vida, 
elaborado pelos pesquisadores com base nas informações contidas na Pesquisa Nacional de Saúde ${ }^{10}$, possui 35 questões com variáveis sociodemográficas (sexo, faixa etária, raça, estado civil, escolaridade, situação ocupacional, renda mensal, situação da moradia, composição familiar), histórico de saúde (tipo de AVC, tempo do ocorrido, fatores de risco e se realiza tratamento), estilo de vida (prática de atividade física, uso de drogas licitas e ilícitas, nível de estresse).

O SF-36 (Medical Outcomes ShortForm 36-item Health Survey) é um questionário multidimensional, de fácil compreensão, traduzido e validado no Brasil $^{11}$. O SF-36 é um questionário reconhecido como "padrão-ouro" para avaliar a qualidade de vida relacionada à saúde (QVRS), servindo como uma escala válida para as condições socioeconômicas e culturais da população brasileira e vem sendo extensamente utilizada em sobreviventes de AVC, permitindo traçar um perfil global das condições funcionais, psicossociais desses pacientes e de suas expectativas em relação à vida ${ }^{12-14}$.

O questionário é composto por 36 itens, subdivididos em oito domínios: 1) Capacidade Funcional (10 itens) avaliando a presença e extensão de limitações relacionadas à capacidade física; 2) Aspectos Físicos (4 itens) avalia as limitações quanto ao tipo e quantidade de trabalho, bem como quanto essas limitações dificultam a realização do trabalho e das atividades da vida diária; 3 ) Dor (2 itens) avalia a presença de dor, sua intensidade e sua interferência nas atividades da vida diária; 4) Estado Geral de Saúde (5 itens) avalia como o paciente se sente em relação à sua saúde global; 5) Vitalidade (04 itens) considera o nível de energia e de fadiga Aspectos Sociais; 6) Aspectos Sociais (02 itens) analisa a integração do indivíduo em atividades sociais; 7) Emocionais (03 itens) avalia o impacto de aspectos psicológicos no bem-estar do paciente e 8) Saúde Mental (05 itens) inclui questões sobre ansiedade, depressão, alterações no comportamento ou descontrole emocional e bem-estar psicológico $^{11,15}$. O SF-36 apresenta um escore final de zero a 100 pontos, sendo que zero corresponde à pior e 100 à melhor percepção da $\mathrm{QV}^{11}$.

Os dados dos questionários foram analisados pela estatística descritiva e inferencial usando programa SPSS Statistic versão 23.0 IBM ${ }^{\circledR}$. Para a análise da distribuição dos dados foi aplicado o Teste de Kolmogorov-smirnov. As variáveis categóricas serão apresentadas por meio da frequência absoluta e relativa e as variáveis numéricas por meio da média \pm desvio padrão (DP).

\section{Resultados}

Durante a realização do estudo, 20 pacientes acometidos por AVC estavam em atendimento nas clínicas CISA e Qualivida, desses apenas 15 atingiram a pontuação necessária no MEEM (20 pontos) para entrar na pesquisa.

Dos 15 pacientes, $9(60,0 \%)$ eram do sexo masculino e $6(40,0 \%)$ do sexo feminino, quanto à idade a maioria tinha idade igual ou superior a 60 anos, com faixas etárias de 60 a 69 anos $6(40,0 \%)$ e 71 a 79 anos 2 (13,3\%). Em relação à raça autorreferida foram predominantes a branca e parda com $7(46,7 \%)$, respectivamente (Tabela 1).

No histórico de saúde observa-se que houve prevalência do tipo AVC isquêmico verificado em $11(73,3 \%)$ dos pacientes e $4(26,7 \%)$ AVC hemorrágico. A percepção dos pacientes em relação à sua saúde no geral é considerada boa para 14 $(93,33 \%)$ pacientes e muito boa apenas para $1(6,67 \%)$. Todos os $15(100 \%)$ pacientes precisaram de ajuda após a alta hospitalar. Já, referente à continuidade da fisioterapia após a alta $10(66,7 \%)$ responderam que não continuaram e $5(33,3)$ que sim. Sobre outras comorbidades presentes nos pacientes, $7(46,7 \%)$ são hipertensos, 5 (33,3\%) são diabéticos e 3 (20\%) possuem alguma doença cardiovascular (Tabela 1).

No que diz respeito ao estilo de vida, referente à prática de atividade física 8 
$(53,3 \%)$ praticam algum tipo de exercício físico, essa prática foi frequente em $3(20 \%)$ realizando $3 x$ por semana, seguido de $2(13,3 \%)$
$2 \mathrm{x}$ por semana e $21 \mathrm{x}$ por semana. Todos os pacientes $15(100 \%)$ relataram não fazer uso de bebida alcoólica e cigarros (Tabela 1).

Tabela 1. Distribuição das variáveis sociodemográficas, histórico de saúde e estilo de vida de pacientes acometidos por AVC, Fortaleza, Ceará, Brasil, 2019.

\begin{tabular}{|c|c|c|}
\hline Variáreis & $\mathbf{n}$ & $\%$ \\
\hline \multicolumn{3}{|c|}{ Variáveis sociodemográficas } \\
\hline \multicolumn{3}{|l|}{ Sexo } \\
\hline Masculino & 9 & 60,00 \\
\hline Feminino & 6 & 40,00 \\
\hline \multicolumn{3}{|l|}{ Faixa-Etária } \\
\hline 30 a 39 & 2 & 13,3 \\
\hline 40 a 49 & 2 & 13,3 \\
\hline 50 a 59 & 3 & 20,0 \\
\hline 60 a 69 & 6 & 40,0 \\
\hline 70 a 79 & 2 & 13,3 \\
\hline \multicolumn{3}{|l|}{ Raça } \\
\hline Branca & 7 & 46,7 \\
\hline Preta & 1 & 6,7 \\
\hline Parda & 7 & 46,7 \\
\hline \multicolumn{3}{|c|}{ Histórico de Saúde e estilo de vida } \\
\hline \multicolumn{3}{|c|}{ Tipo de AVC } \\
\hline AVC Isquêmico & 11 & 73,3 \\
\hline AVC Hemorrágico & 4 & 26,6 \\
\hline \multicolumn{3}{|l|}{ Estado de saúde geral } \\
\hline Ruim & 0 & 0 \\
\hline Boa & 14 & 93,3 \\
\hline Muito boa & 1 & 6,6 \\
\hline \multicolumn{3}{|l|}{ Ajuda após alta } \\
\hline Sim & 15 & 100,0 \\
\hline Não & 0 & 0 \\
\hline \multicolumn{3}{|c|}{ Ao sair continuou a fisioterapia } \\
\hline $\operatorname{Sim}$ & 5 & 33,3 \\
\hline Não & 10 & 66,7 \\
\hline \multicolumn{3}{|l|}{ Tem HAS } \\
\hline $\operatorname{Sim}$ & 7 & 46,7 \\
\hline Não & 8 & 53,3 \\
\hline \multicolumn{3}{|l|}{ Tem Diabetes } \\
\hline Sim & 5 & 33,3 \\
\hline Não & 10 & 66,7 \\
\hline \multicolumn{3}{|l|}{ Tem DCV } \\
\hline Sim & 3 & 20,0 \\
\hline Não & 12 & 80,0 \\
\hline \multicolumn{3}{|c|}{ Pratica atividade física } \\
\hline Sim & 8 & 53,3 \\
\hline Não & 7 & 46,7 \\
\hline \multicolumn{3}{|l|}{ Quantas vezes na semana } \\
\hline 1x Por semana & 2 & 13,3 \\
\hline 2x Por semana & 2 & 13,3 \\
\hline $3 x$ Por semana & 3 & 20,0 \\
\hline Mais de $3 x$ por semana & 1 & 6,7 \\
\hline \multicolumn{3}{|l|}{ Uso de bebida alcoólica } \\
\hline Sim & 0 & 0 \\
\hline Não & 15 & 100 \\
\hline \multicolumn{3}{|l|}{ Uso de cigarro } \\
\hline $\operatorname{Sim}$ & 0 & 0 \\
\hline Não & 15 & 100 \\
\hline
\end{tabular}

Fonte: Autores, 2019. 
$\mathrm{Na}$ análise do questionário SF-36, o maior comprometimento ocorreu nos domínios: Aspectos físicos, emocionais e capacidade funcional, além destes os demais apresentaram valores considerados baixos. No entanto o domínio aspectos sociais (AS) apresentou os maiores valores com escore médio de 66,60 $( \pm 21,29)$ (Tabela 2).

Tabela 2. Distribuição dos domínios do questionário SF-36. Fortaleza, Ceará, Brasil, 2019.

\begin{tabular}{cc}
\hline Domínios SF36 & Média \pm DP \\
\hline CF & $43,33 \pm 26,70$ \\
AF & $26,66 \pm 6,45$ \\
DOR & $58,53 \pm 20,39$ \\
EGS & $59,53 \pm 35,98$ \\
VITALIDADE & $60,33 \pm 18,65$ \\
AS & $66,60 \pm 21,29$ \\
AE & $41,93 \pm 23,57$ \\
SM & $62,40 \pm 11,39$ \\
\hline
\end{tabular}

$\mathrm{CF}=$ Capacidade Funcional; $\mathrm{AF}=$ Aspectos Físicos; $\mathrm{D}=$ Dor; EGS=Estado Geral de Saúde; V=Vitalidade; $\mathrm{AS}=$ Aspectos Sociais; $\mathrm{AE}=$ Aspectos Emocionais; $\mathrm{SM}=$ Saúde Mental.

Fonte: Autores, 2019.

\section{Discussão}

A presente pesquisa buscou analisar a qualidade de vida dos pacientes acometidos por acidente vascular cerebral. Considerada uma Doença Crônica Não Transmissível (DCNT), o AVC gera inúmeras limitações de ordem neurológica que comprometem a capacidade física e emocional dos indivíduos. Dentre as limitações, destacam-se as alterações funcionais e cognitivas, além de desencadear o aparecimento de sintomas depressivos, que interferem de forma parcial ou total na realização das atividades de autocuidado, laborais e vida diária, afetando a QV do paciente ${ }^{6,16}$.

Em relação às variáveis sociodemográficas, verificou-se maior proporção no sexo masculino $60 \%$, com idade igual ou superior a 60 anos, sendo predominante na amostra a cor branca e parda. Diante disso, sugere-se que com o envelhecimento humano a probabilidade de ocorrer um AVC é maior devido a uma série de fatores contextuais como, o estilo de vida e comorbidades, consequentemente os idosos estão mais sujeitos ao acometimento da doença do que indivíduos mais jovens. Porém, a incidência da doença pode ocorrer em qualquer fase da vida, independente do sexo e cor.

Pesquisas realizadas no Brasil corroboram com os resultados do perfil sociodemográfico encontrados nesta pesquisa. De acordo com os estudos como os de Barella el al. ${ }^{17}$, Araújo et al. ${ }^{3}$, Da Silva et al. ${ }^{18}$ e Pacheco et al. ${ }^{19}$, constataram-se que a faixa etária dos pacientes acometidos pelo AVC ocorreu acima de 60 anos, sendo maior no sexo masculino com cor predominante branca e parda. No entanto, Carvalho e Deodato ${ }^{20}$, verificaram em seu estudo a prevalência do AVC no sexo feminino, porém as variáveis idade e cor foram semelhantes aos dados encontrados nesta pesquisa.

O tipo de AVC é definido de acordo com a etiologia da lesão. Segundo Asplund et $a l^{2}{ }^{2}$ a classificação pode ser em AVCi e $\mathrm{AVCh}$, além destas a doença pode ocorrer com um quadro transitório, caracterizado por uma lesão isquêmica focal com rápida duração e reversão completa do quadro em até 24 horas. Os resultados desta pesquisa são condizentes com a literatura, verificando que o AVCi ocorreu em $73,3 \%$ da amostra. Visto que estudos 
epidemiológicos apontam que o maior número de casos é do tipo isquêmico ${ }^{17,21,22}$.

No mundo, o AVC ocupa o segundo lugar como principal causa de morte, tendo maior frequência em adultos e idosos. No Brasil, devido às mudanças no perfil de morbimortalidade nos últimos anos, o AVC como uma DCNT, é responsável por um elevado índice de internações e mortalidade ${ }^{23}$. Segundo Langhorne et al. ${ }^{24} \mathrm{o}$ AVCi apresenta maior taxa de incidência e morbidade, ocorrendo em $80 \%$ dos casos. As sequelas físicas e psicológicas que limitam as realizações de AVDs, tornam o paciente dependente de um cuidador durante o tratamento e em muitos casos por toda a vida.

Nosso estudo verificou que toda a amostra obteve ajuda familiar e/ou cuidadores após a alta hospitalar. Para Ovbiagele et $a l .{ }^{25}$, no momento em que os pacientes retornam aos seus domicílios, deparam-se com novas barreiras como a realização das AVD's, que antes eram realizadas com independência, porém após o AVC as tarefas tornaram-se dependentes de auxílios específicos, o que dificulta a realização destas.

$\mathrm{O}$ apoio familiar em conjunto com uma abordagem multidisciplinar contribui para uma melhora da QV dos pacientes. Dentre as condutas de tratamento, a reabilitação fisioterapêutica é de suma importância para a recuperação funcional, independência e autonomia, que consequentemente interfere de forma positiva nos aspectos biopsicossociais da população estudada ${ }^{26}$. No entanto, $66,7 \%$ dos pacientes deste estudo interromperam o tratamento fisioterapêutico após alta hospitalar. Contudo, esse dado pode estar relacionado às limitações funcionais $\mathrm{e}$ condições socioeconômicas vividas pela amostra.

Referente às comorbidades que predispõem o AVC, constatou-se na amostra que $7(46,7 \%)$ tinham HAS, 5 $(33,3 \%)$ tinham diabetes e apenas $3(20 \%)$ tinham DCV. Em relação aos hábitos de vida, $15(100 \%)$ não fazem uso de bebidas alcoólicas e cigarros, quanto à prática de exercícios físicos, $8(53,3 \%)$ praticam algum tipo de atividade física pelo menos 3 vezes na semana.

Os fatores de risco modificáveis como: estresse, hipertensão, diabetes, tabagismo, etilismo, obesidade e doenças cardiovasculares, são descritos pela literatura como comorbidades favoráveis para o acometimento de doenças cerebrovasculares, principalmente $\mathrm{o}$ $\mathrm{AVC}^{25,27,28}$. No entanto, estudos apontam que aderir a um estilo de vida saudável como uma boa alimentação e práticas de atividades físicas contribui de forma significativa para manter os níveis adequados de autonomia motora, melhora da saúde física, mental e socialização ${ }^{19,29}$. Portanto, a reincidência da doença diminui assim como a incidência de novos casos.

No presente estudo foram avaliados os fatores referentes aos aspectos biopsicossociais, que comprometem as condições de saúde e QV dos indivíduos acometidos pelo AVC. Os escores obtidos pelo SF-36, que é padrão ouro para avaliar a QV relacionada à saúde, mostraram que todos os domínios do referido instrumento foram comprometidos do maior ao menor grau, respectivamente: $\mathrm{AF}(26,76), \mathrm{AE}$ (41,93), CF $(43,33)$, D $(58,53)$, EGS $(59,53)$, V $(60,33)$, SM $(62,40)$ e AS $(66,60)$.

Considerando os 8 domínios avaliados pelo SF-36, a capacidade funcional e os aspectos físicos avaliam a presença de limitações que prejudicam a realização de atividades de autocuidado, trabalho, entre outras. A dor é verificada quanto sua presença, intensidade e interferência nas AVDs, enquanto o estado geral de saúde avalia como o paciente se sente em relação à sua saúde global. Os aspectos sociais e emocionais analisam a socialização, impactos psicológicos no bem-estar do paciente e a saúde mental aborda questões sobre ansiedade, depressão, alterações no comportamento ou descontrole emocional. Dessa forma, o 
instrumento possibilita a análise da QV por uma visão multidimensional.

Estudos realizados no Brasil encontraram resultados semelhantes ao desta pesquisa no que concerne aos escores do SF-36. Scalzo et al. ${ }^{30}$, verificaram em sua amostra os seguintes escores: $\operatorname{AF}(34,1)$, capacidade funcional $(37,8), \mathrm{AE}(53,9)$, dor $(60,5)$, EGS $(62,8)$, saúde mental $(62,9)$, vitalidade $(67,1)$ e por último, AS $(76,8)$. Da mesma forma outro estudo realizado por Silva $^{31}$, obteve achados similares sendo a CF $(44,72)$, AF $(44,40)$, EGS $(62,08), \mathrm{V}$ $(78,61)$, AS $(62,50)$, AE $(50,00)$ SM $(73,56)$.

Os aspectos relacionados à capacidade física e o desempenho funcional mensurados neste estudo e presentes na literatura, podem ser entendidos diante das limitações como vestir-se, banhar-se e até mesmo caminhar pequenas distâncias de forma independente gerando déficit nesses pacientes, sendo um fator importante na percepção da $\mathrm{QV}^{28}$. Os aspectos emocionais estão relacionados a dificuldades em seu âmbito domiciliar, nas suas AVD's e principalmente no trabalho, o que pode gerar afastamento provisório ou definitivo por invalidez, isso é um fator que contribui para o baixo escore no domínio, corroborando com os dados da presente pesquisa $^{30}$.

A análise da qualidade sempre será um desafio e um campo abrangente, pois trata-se de algo subjetivo e particular de cada indivíduo. Nesse contexto, as condições de saúde e de vida das pessoas pós-AVC, tendem a declinar por conta das limitações físicas e emocionais impostas pela doença. No entanto, os fatores de risco que predispõem a incidência e reincidência de tal patologia, podem ser evitados mediante as práticas de hábitos saudáveis como boa alimentação e prática de atividades físicas, sendo necessário conscientização e iniciativa por parte do poder público bem como da sociedade em geral.

Ademais, são reconhecidas algumas limitações neste estudo como a amostra por conveniência, o que resulta no número de participantes e locais de pesquisa limitados, isso dificulta a generalização dos resultados; outro ponto limitante foi a não utilização de um questionário específico sobre QV em pacientes com AVC. No entanto, os resultados deste estudo são relevantes pois sugerem que indivíduos acometidos por AVC passam a ter prejuízos em sua qualidade de vida. Assim, espera-se que nossos achados contribuam para a discussão do tema e incentive a realização de novas pesquisas com maiores amostras.

\section{Conclusão}

Foi observado o comprometimento na percepção da $\mathrm{QV}$ com valores baixos de todos os domínios e principalmente nos aspectos físicos, emocionais e na capacidade funcional que gera uma baixa $\mathrm{QV}$, com maior frequência de pacientes com diagnóstico clínico de AVC do tipo isquêmico, sendo predominante em indivíduos com idade igual ou superior a 60 anos, do gênero masculino.

Diante disso, apesar das limitações encontradas neste estudo, os resultados desta pesquisa são relevantes para a temática estudada, podendo estimular e nortear novas práticas de assistência, ampliando a prevenção de doenças e incentivando ações de promoção de saúde para melhorar o bem-estar da população estudada. Contudo, sugere-se a realização de mais pesquisas com amostras maiores para que se possa identificar e minimizar os fatores que interferem na $\mathrm{QV}$ dos pacientes acometidos por AVC.

\section{Referências}

1. World Health Organisation. A clear message emerges from this report: unless immediate action is taken globally, the neurological burden is likely to become an even 
more serious threat to public health. World Heal Organ. 2015. https://www.who.int/mental_health/neurology/chapter_4_neuro_disorders_public_h_c hallenges.pdf

2. Asplund K, Sukhova M, Wester P, Stegmayr B. Diagnostic procedures, treatments, and outcomes In stroke patients admitted to different types of hospitals. Stroke. 2015;46(3):806-12.

https://www.ahajournals.org/doi/pdf/10.1161/STROKEAHA.114.007212

3. Araújo JP, Darcis JVV, Tomas AVC, Centro. Tendência da Mortalidade por Acidente Vascular Cerebral no Município de Maringá, Paraná entre os Anos de 2005 a 2015. Int J Cardiovasc Sci. 2017;50(18):590-1. http://dx.doi.org/10.5935/2359-4802.20170097

4. Castro HHG, Alencar AP, Benseñor IM, Lotufo PA, Goulart AC. Multimorbidities are associated to lower survival in Ischaemic stroke: Results from a Brazilian stroke cohort (EMMA Study). Cerebrovasc Dis. 2017;44(3-4):232-9. https://doi.org/10.1159/000479827

5. Fleck MPA, Lousada S, Xavier M, Chachamovich E, Vieira G, Santos L, et al. Aplicação da versão em português do instrumento de avaliação de qualidade de vida da Organização Mundial da Saúde (WHOQOL-100). Rev Saude Publica. 1999;33(2):198205. https://doi.org/10.1590/S0034-89101999000200012

6. Canuto MÂ, Nogueira LT. Stroke and quality of life: an integrative review. Rev Pesqui Cuid é Fundam Online. 2015;7(2):2561-8. https://doi.org/10.9789/21755361.2015.v7i2.2561-2568

7. Rangel ESS, Belasco AGS, Diccini S. Qualidade de vida de pacientes com acidente vascular cerebral em reabilitação. ACTA Paul Enferm. 2013;26(2):205-12. https://doi.org/10.1590/S0103-21002013000200016

8. Bertolucci PHF, Brucki SMD, Campacci SR, Juliano Y. O Mini-Exame do Estado Mental em uma população geral: impacto da escolaridade. Arq Neuropsiquiatr. 1994;52(1):01-7. https://doi.org/10.1590/s0004-282x2003000500014

9. Brucki SMD, Nitrin R, Caramelli P, Bertolucci PHF, Okamoto IH. Suggestions for utilization of the mini-mental state examination in Brazil. Arq Neuropsiquiatr. 2003;61(3 B):777-81. https://www.scielo.br/pdf/anp/v61n3B/17294.pdf

10. Moreira C. Ciclos de vida. Vol. 2, Instituto Brasileiro de Geografia e Estatística - IBGE. 2014. catalogo? view $=$ detalhes\&id $=525$

https://biblioteca.ibge.gov.br/index.php/biblioteca-

11. Ciconelli RM, Ferraz MB, Santos W, Meinão I, Quaresma MR. Tradução para a língua portuguesa e validação do questionário genérico de avaliação de qualidade de vida SF36 (Brasil SF-36) [Internet]. Vol. 39, Revista Brasileira De Reumatologia. 1999. p. 14350 .

https://www.ufjf.br/renato_nunes/files/2014/03/Valida\%25C3\%25A7\%25C3\%25A3odo-Question\%25C3\%25A1 rio-de-qualidade-de-Vida-SF-36.pdf

12. Mayo NE, Wood-Dauphinee S, Côté R, Durcan L, Carlton J. Activity, participation, and quality of life 6 months poststroke. Arch Phys Med Rehabil. 2002;83(8):1035-42. http://dx.doi.org/10.1053/apmr.2002.33984

13. Makiyama TY, Battisttella LR, Litvoc J, Martins LC. Estudo sobre a qualidade de vida de pacientes hemiplégicos por acidente vascular cerebral e de seus cuidadores. Acta Fisiátrica [Internet]. 2004;11(3):106-9. http://dx.doi.org/10.5935/0104-7795.20040004

14. Patel MD, Tilling K, Lawrence E, Rudd AG, Wolfe CDA, McKevitt C. Relationships between long-term stroke disability, handicap and health-related quality of life. Age Ageing. 2006;35(3):273-9. http://dx.doi.org/10.1093/ageing/afj074

15. De Oliveira MR, Orsini M. Quality of life scales for Brazilian stroke patients. Rev Neurociencias. 2009;17(3):255-62. https://doi.org/10.34024/rnc.2009.v17.8550 
16. Ramos-Lima MJM, Brasileiro I de C, de Lima TL, Braga-Neto P. Quality of life after stroke: Impact of clinical and sociodemographic factors. Clinics. 2018;73:1-7. https://doi.org/10.6061/clinics/2017/e418

17. Barella RP, Duran V de AA, Pires AJ, Duarte R de O. Perfil do atendimento de pacientes com acidente vascular cerebral em um hospital filantrópico do sul de santa catarina e estudo de viabilidade para implantação da unidade de AVC. Arq Catarinenses Med [Internet].

2019;48(1):131-43. http://www.acm.org.br/acm/seer/index.php/arquivos/article/view/432/334

18. Da Silva PLN, Dos Santos LS, Ribeiro DDD, Guimarães HDG, Esteves KAF, Alves ECS, et al. Análise da prevalência de acidente vascular encefálico em pacientes assistidos por uma instituição hospitalar. JMPHC | J Manag Prim Heal Care. 2018;9:115. https://doi.org/10.14295/jmphc.v9i0.333

19. Pacheco FT, Littig IA, Gagliardi RJ, Da Rocha AJ. Multidetector computed tomography angiography in clinically suspected hyperacute ischemic stroke in the anterior circulation: An etiological workup in a cohort of Brazilian patients. Arq Neuropsiquiatr. 2015;73(5):408-14. https://doi.org/10.1590/0004-282X20150034

20. Carvalho IA. Fatores de risco do acidente vascular encefálico. Rev Científica da FASETE. 2016;2(1):180-94. https://doi.org/10.16891/2317.434X.134

21. Ribeiro K, Neves R, Brito G, Morais J, Lucena E, Medeiros J. Perfil de Usuários Acometidos por Acidente Vascular Cerebral Adscritos à Estratégia Saúde da Família em uma Capital do Nordeste do Brasil. Rev Bras Ciências da Saúde. 2012;16(2):35-44. https://doi.org/ 10.4034/rbcs.2012.16.s2.05

22. Carvalho MIF, Delfino JAS, Pereira WMG, Matias EFSS. Acidente vascular cerebral: dados clínicos e epidemiológicos de uma clínica de fisioterapia do sertão nordestino brasileiro. Rev Interfaces Saúde, Humanas e Tecnol. 2015;2(6):1-6. http://dx.doi.org/10.16891/2317.434X.134

23. Almeida SRM. Análise epidemiológica do acidente vascular cerebral no Brasil. Rev Neurociencias. 2012;20(4):481-2. doi: https://doi.org/10.4181/RNC.2012.20.483ed.2p

24. Langhorne P, Bernhardt J, Kwakkel G. Stroke rehabilitation. Lancet [Internet]. 2011;377(9778):1693-702. https://doi.org/10.1016/S0140-6736(11)60325-5

25. Ovbiagele B, Bath PM, Cotton D, Vinisko R, Diener HC. Obesity and recurrent vascular risk after a recent ischemic stroke. Stroke. 2011;42(12):3397-402. https://doi.org/10.1161/STROKEAHA.111.624957

26. Mendonça LB de A, Lima FET, Oliveira SKP de. Acidente vascular encefálico como complicação da hipertensão arterial: quais são os fatores intervenientes? Esc Anna Nery. 2012;16(2):340-6. https://doi.org/10.1590/s1414-81452012000200019.

27. Martins EDRC. Estudo epidemiológico sobre Acidente Vascular Encefálico em uma Clínica Escola de Fisioterapia. Rev saúde pública do Paraná.2016;17(1):32-8. http://www2.unicentro.br/defisio/files/2015/07/Estudoepidemiol\%C3\%B3gico-sobreAcidente-Vascular-Encef\%C3\%A1lico-em-uma-Cl\%C3\%ADnica-Escola-deFisioterapia.pdf

28. Costa TF da, Gomes TM, Viana LR de C, Martins KP, Costa KN de FM. Acidente vascular encefálico: características do paciente e qualidade de vida de cuidadores. Rev Bras Enferm. 2016;69(5):933-9. https://doi.org/10.1590/0034-7167-2015-0064

29. Andersen KK, Olsen TS. The obesity paradox in stroke: Lower mortality and lower risk of readmission for recurrent stroke in obese stroke patients. Int J Stroke. 2015;10(1):99104. https://doi.org/10.1111/ijs.12016

30. Scalzo PL, De Souza ES, Moreira AGDO, Vieira DAF. Qualidade de vida em pacientes com acidente vascular cerebral: Clínica de fisioterapia Puc Minas Betim. Rev Neurociencias. 2010;18(2):139-44. https://doi.org/10.34024/rnc.2010.v18.8480 
31. Gabriele Tainá da Silva F de OY. Dor, desempenho funcional e qualidade de vida após o acidente vascular encefálico. Cent Univ do Sul Minas. 2017;1(1):1-37. http://192.100.247.84/bitstream/prefix/1128/1/TCC_ves\%c3\%a3ofinal_Gabi\%2004.1 1.17.pdf

\section{Como citar este artigo:}

Silva FVM, Oliveira ABC, Brito CB, Sousa FDS, Maia EM, Silva JVP, Ferreira WSB, Nunes PPB. Qualidade de vida de pacientes acometidos por acidente vascular cerebral. Rev. Aten. Saúde. 2021; 19(69): 317-327. 